\title{
MANUFACTURER ORIENTED MIXED MODEL ASSEMBLY LINE SCHEDULING USING DYNAMIC PROGRAMMING
}

\author{
ZAHEER AHMAD $^{1}$, SHAHID YOUSAF $^{2}$ \\ ${ }^{1}$ I.T Department, Govt. College of Commerce, Shahdra, Lahore, \\ ${ }^{2}$ Department of Computer Science, University of Lahore \\ zaheer190@gmail.com,shahid.yousaf@cs.uol.edu.pk
}

\begin{abstract}
Assembly lines have very important role in manufacturing. These are used to manufacture large scale series of products. Developments over the period of time changed the assembly lines from single model lines to more convenient systems with some variations including two assembly lines, ' $n$ ' assembly lines, customer oriented mixed model, multi model and $u$ shaped lines and many more. In this paper attention is focused to develop the model which is used to assemble the three different types of autos which have some common auto parts. The main objective is to design this model to reduce the time when the different types of autos having some common auto parts are assembled. The optimization problem in this paper is solved by using dynamic programming approach. For this purpose six assembly lines are used having ' $n$ ' number of stations, two assembly lines are specified for each type of auto. Assembly lines are set in such a way that the common auto part of three types must be assembled at the parallel station of the lines.
\end{abstract}

Keywords: Optimization problem, Dynamic programming, Manufacturer oriented mixed model, assembly line scheduling.

\section{Introduction}

In the early 1900s a method of production, known as assembly line, made possible the faster and cheaper production of the goods, this concept moved towards the automobile industries. Assembly line has several workstations in a sequence and a specific set of tasks is performed on each of them. The movement of the product is according to their order, through the line from one workstation to next workstation [1]. In addition, assembly lines are used to manufacture variety of products and now a day the assembly line is very attractive way for production [8]. Assembly lines play very important role in the field of manufacturing problems and make possible to develop a product faster and the cost of the product can be decreased.

There are lots of applications where the assembly lines are applicable, e.g., the assembly lines are well known in multiprocessor scheduling area. Assembly lines have been used to develop the automated manufacturing of process control board (PCB) in [6]. Toshiba's compressors of different types (air condition and refrigerant) are assembled by using mixed model assembly line scheduling to fulfill the complicated component supply requirement [3]. Robotic assembly line balancing (RALB) algorithm [5] has been used to balance the assembly line by assigning the most efficient robot types of robot when there are different types of robot to assemble. Assembly lines have also been applied in networking and the idea of the protocol "bucket brigades" has been implemented in in-tree assembly network [7]. In subassembly line's network it is difficult to balance the assembly line as it is required [7]. Generally, problems can be divided into the sub-problems where the assembly lines can be used in any of the i.e. simple assembly lines. U shaped assembly line and mixed model assembly line etc. 
Manufacturer oriented mixed model assembly line scheduling is purposed in the literature by using dynamic programming approach. Further, two assembly line scheduling algorithm is developed in [2] however this algorithm is only for one type of auto and only two assembly lines. Mixed model assembly line scheduling has also been developed by using lagrangian relaxation technique and this model is under the development of Toshiba [3].

The proposed model in this paper is especially design for three types of autos which have some common auto parts. For this model six assembly lines are used two lines for each type. It is defined that if the auto is of type 1 then it can enter either from line1 or line2. Similarly for the autos of type2, line 3 or the line 4 is used for the purpose of entrance and for the type 3 autos they can enter from line 5 or line6. Then the stations of all six lines are examined if the work done on the stations are same (i.e. the type of auto parts of autos is assembled at these stations is same) then it can be moved to any of the six line where the minimum assembling time is required. This procedure will continue till the $n t h$ station.

The assembly line can be operated manually or it can be automated. Initially the manual assembly line was made and it was much faster than the work done in unordered format. Later on the automated assembly line came into being which was faster than the manual assembly line and much faster than the unordered work format. But in the automated we have to spend a big investment for dedicated resources. The assembly line is the efficient procedure to manufacture the products. For assembly line task when flexible equipment is used the designing issue of an assembly line is very important [5].

The paper is organized as follows. In section 2, application of assembly lines are discussed. In section 3, survey of some existing work is explored. A problem definition is discussed in section 4 and in section 5 mathematical model is described and then finally the conclusion and future works in section 6.

\section{Survey of Existing Work}

Researcher always working for development in different areas of research, due to this work new methods and models come into being at different time. So on assembly lines many researchers have developed the model and algorithms at different times. Some of them are describes below. Joseph Bukchin and Michal Tzur [1] develop a new method of flexible assembly line when several types of equipment are available at assembly lines by using the branch and bound technique. The purpose of it is to minimize the total equipment cost. At School of Industrial and Systems Engineering, Georgia Institute of Technology [10] the concept of "bucket brigade" which is a protocol is introduce on in-tree assembly network. The idea of a supporting tool for manufacturing system [9] simulation and gamming is given by Durk-Jouke van der Zee Jannes Slomp. The objective of this development is to minimize the associative cost for the design of assembly line by providing a comprehensive approach. Generalized flexible line balancing (GFLB) is used to manufacture the printed circuit board. This concept is given by the Timo H"ayrinen, Mika Johnsson, Tommi Johtela, Jouni Smed and Olli Nevalainen in 1998 [11]. Mixed assembly line scheduling [3] has also developed by using the lagrangian relaxation technique. This model used to assemble Toshiba's compressor i.e. air condition and refrigerant equipment. The objective of this model is to determined significant line and in a sequence for all lots so that they are delivered on due dates by avoiding starvation of component. To design and balance robotic assembly line heuristic approach is used [5]. To make the efficient use of assembly line it must be balanced. The objective of it is to balance the assembly line. Emanuel Falkenauer [12] said that the line balancing is utilized in different problems. He describes different ways of assembly line in the article. Concurrent operation and parallel machines are also handled by assembly line scheduling [7]. The efficient use of the concurrent operations is possible by implementing this scheduling and by preventing the assembly lines from degenerating into serially performing of operations, first construct a scheduler of single machine where each concurrent operations are performed then the generalized scheduler for concurrent operation which are perform on identical set of parallel machines.

\section{Solution Methodology of the Model}

The dynamic programming is well known approach, and is applicable when the sub problem is not independent. Typically there are four steps of dynamic programming .the first step; character the structure of an optimal solution is performed in this model is as fellows. The fastest possible way for auto part to get from the starting point through the station $S_{i, j}$ if the type of auto is 1 then it can enter either from line1 or line 2 if the type is 2 then it can enter either from line 3 or line 4 and if the type of auto is 3 then it can enter either from line 5 or line6.and reached at the station 
$\mathrm{S}_{\mathrm{i}, 1}$ (ie $1^{\text {st }}$ station of any of the six line). when $\mathrm{j}=2,3,4 \ldots \ldots . \mathrm{n}$ then it check the conditions if the auto part assemble on the $\mathrm{S}_{\mathrm{i}, \mathrm{j}}$ of six lines is same the it can came any of the six line .if the assembling auto part is same on the four line then it came from any of the four lines if there is no common auto part assembled the it came from only two lines ie from selected lines which are pre mentioned

The second step of the dynamic programming is to define the value of an optimal solution recursively in term of optimal solutions to sub problems. The fastest way through stations when $\mathrm{j}=1.2 \ldots . \mathrm{n}$ and we suppose fi[j] is the fastest possible time to get auto part from starting point through station si,j

The fastest time denoted by $\mathrm{f}^{*}$ it is obtained by entry time through the station $\mathrm{n}$ either of six line and then exit time $f^{*}=(\min (f 1[n]+x 1, f 2[n]+x 2, f 3[n]+x 3, f 4[n]+x 4, f 5[n]+x 5, f 6[n]+x 6))$

When the type is 1 then to get though the station 1 on either of first two line, an auto part just goes directly through the station.

$\mathrm{fi}[1]=\mathrm{e} 1+\mathrm{a} 1,1$ and $\mathrm{f} 2[1]=\mathrm{e} 2+\mathrm{a} 2,1$

When the type is 2 then to get though the station 1 on either of line 3 and line 4 , an auto part just goes directly through the station.

$\mathrm{f} 3[1]=\mathrm{e} 3+\mathrm{a} 3,1$ and $\mathrm{f} 2[1]=\mathrm{e} 4+\mathrm{a} 4,1$

When the type is 3 then to get though the station 1 on either of line 5 and line6, an auto part just goes directly through the station.

$f 5[1]=e 5+a 5,1$ and $f 6[1]=e 6+a 6,1$

When the $j=2,3,4 \ldots \ldots \ldots \ldots \ldots . . . n$ then for:

$\mathrm{f} 1[\mathrm{j}]$ is computed as

$$
\begin{array}{rc}
f 1[j]=\min (f 1[j-1]+a i, j+t 1, j-1,1, & f 2[j-1]+a i, j+t 2, j-1,1 \\
f 3[j-1]+a i, j+t 3, j-1,1 ; & f 4[j-1]+a i, j+t 4, j-1,1 \\
f 5[j-1]+a i, j+t 5, j-1,1, & f 6[j-1]+a i, j+t 6, j-1,1)
\end{array}
$$

$\mathrm{f} 2[\mathrm{j}]$ is compute as

$$
\begin{array}{rc}
f 2[j]=\min (f 1[j-1]+a i, j+t 1, j-1,1, & f 2[j-1]+a i, j+t 2, j-1,1 \\
f 3[j-1]+a i, j+t 3, j-1,1 ; & f 4[j-1]+a i, j+t 4, j-1,1 \\
f 5[j-1]+a i, j+t 5, j-1,1, & f 6[j-1]+a i, j+t 6, j-1,1)
\end{array}
$$

$\mathrm{f} 3[\mathrm{j}]$ is compute as

$$
\begin{array}{rc}
f 3[j]=\min (f 1[j-1]+a i, j+t 1, j-1,1, & f 2[j-1]+a i, j+t 2, j-1,1 \\
\text { f3 [j-1]+ai,j t t3, j-1,1; } & f 4[j-1]+a i, j+t 4, j-1,1 \\
\text { f5 }[j-1]+a i, j+t 5, j-1,1 & \text { f6 }[j-1]+a i, j+t 6, j-1,1)
\end{array}
$$

$\mathrm{f} 4[\mathrm{j}]$ is compute as

$$
\begin{aligned}
f 4[j]=\min (f 1[j-1]+a i, j+t 1, j-1,1, & f 2[j-1]+a i, j+t 2, j-1,1 \\
f 3[j-1]+a i, j+t 3, j-1,1 ; & f 4[j-1]+a i, j+t 4, j-1,1 \\
f 5[j-1]+a i, j+t 5, j-1,1 & f 6[j-1]+a i, j+t 6, j-1,1)
\end{aligned}
$$

$\mathrm{f} 5[\mathrm{j}]$ is compute as

$$
\begin{array}{rc}
f 5[j]=\min (f 1[j-1]+a i, j+t 1, j-1,1, & f 2[j-1]+a i, j+t 2, j-1,1 \\
f 3[j-1]+a i, j+t 3, j-1,1 ; & f 4[j-1]+a i, j+t 4, j-1,1 \\
f 5[j-1]+a i, j+t 5, j-1,1, & f 6[j-1]+a i, j+t 6, j-1,1) \\
\text { compute as } & \\
\text { f5[j]= min (f1 } \begin{aligned}
{[j-1]+a i, j+t 1, j-1,1, } & f 2[j-1]+a i, j+t 2, j-1,1 \\
f 3[j-1]+a i, j+t 3, j-1,1 ; & f 4[j-1]+a i, j+t 4, j-1,1
\end{aligned}
\end{array}
$$

$\mathrm{f} 6[\mathrm{j}]$ is compute as 


$$
f 5[j-1]+a i, j+t 5, j-1,1, \quad f 6[j-1]+a i, j+t 6, j-1,1)
$$

\section{Problem Definition}

Which one stations should be chosen to minimize the assembly time when three different types of autos are assembled and we have just six assembly lines, two assembly lines for each type of auto and these three types of auto must have some common auto parts.

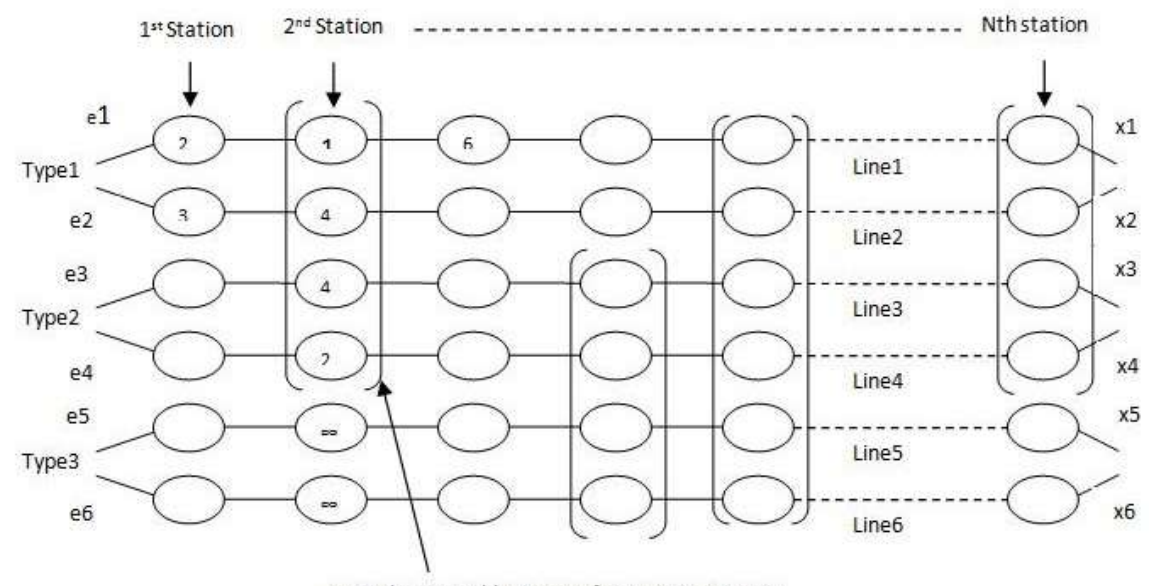

Here the assembling type of spare part is same

Fig. 1 Circle shows the station and e1 is entry time for line 1 and similarly e2, e3, e4, e5, e6 are respectively for line2,line3, line4,line5 and for line $6, \mathrm{x} 1, \mathrm{x} 2, \mathrm{x} 3, \mathrm{x} 4, \mathrm{x} 5, \mathrm{x} 6$ is exit time the circle in the square bracket shows that her the assembling auto part is same.

This model is design to minimize the assembly time when three different types of autos are assembled and we have just six assembly lines, two assembly lines for each type of autos and these three autos have some common auto parts. Let us take the following figure for explanation.

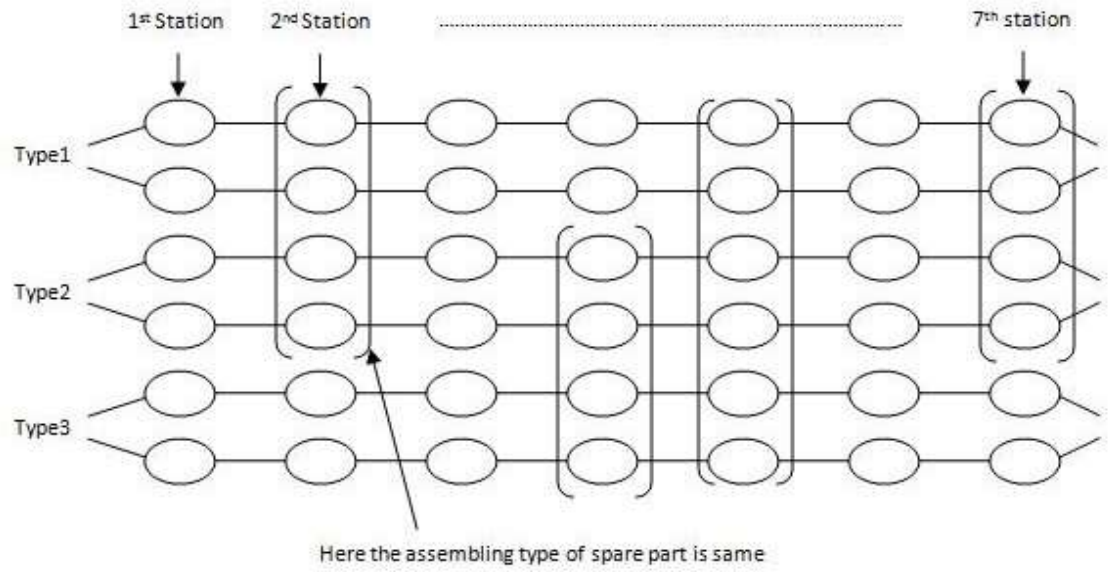

Fig. 2 Three are six (6) assembly lines first two lines are for type 1 and $3^{\text {rd }}$ and $4^{\text {th }}$ lines are for type 2 of auto and $5^{\text {th }}$ and $6^{\text {th }}$ line are for type 3 of auto and there are only 7 numbers of stations. The figure show that at the station 2 the auto parts assemble is same for both type 1 and type 2 of autos. Suppose the auto is of type 1 and enter from either line 1 or line 2 then according to the fig 1 it can move to the station 2 of any of the first four lines where it take minimum time. then at $3^{\text {rd }}$ station and $4^{\text {th }}$ there are no common auto part so here it works just like a two assembly lines[3] scheduling then at the $5^{\text {th }}$ station the auto part assemble at each line is same so calculate assembly time of 
all the station and the minimum is chosen and then at the 6th station no common auto parts are assemble so calculate the station line 1 and line 2 and other are consider as infinite $f 3[6]=\infty \quad f 4[6]=\infty \quad f 5[6]=\infty$ and $f 6[6]=\infty$.

\section{Mathematical Model}

Now the mathematical model of the it is given, we can say that this model is a variation of two assembly line[3] because its internal work is just like a two assembly line scheduling

\section{Assumptions in this model}

This model has some assumptions and limitations which are given below.

1. There are six assembly lines each with $\mathrm{n}$ stations.

2. There are three types of autos.

3. Two assembly lines for each type of autos.

4. The jth station on line is denoted by $\mathrm{Si}, \mathrm{j}$.

5. The assembly time at that station is ai, $\mathrm{j}$.

6. An auto enters factory, goes into line I taking time ei.

7. After going through $\mathrm{jth}$ station on line $\mathrm{i}$, the auto goes to the $(j+1)$ stp station on any line where assembling type is same.

8. There is also transfer cost if it stays on the same line.

9. It takes time ti,j to transfer to the other line after station Si.j.

10. $\mathrm{L}$ is that line to which transfer is made.

11. After exiting the nth station on a line, it takes time xi for the completed auto to exit the factory.

Problem is to determine which stations to choose from line 1 to line $\mathrm{n}$ to minimize total time through the factory.

\section{Base case:}

The base case of this model is

$\left.\begin{array}{l}\mathrm{f} 1[1]=\mathrm{e} 1+\mathrm{a} 1,1 \\ \mathrm{f} 2[1]=\mathrm{e} 2+\mathrm{a} 2,1\end{array}\right\} \quad$ When Type $=1$

If the auto type is 1 then it can be enter either from the line 1 or from the line2.

f1 [1] is a total time at station number 1 of the line 1, including the entry time e1 and the assemble time a1, 1 at station 1. Similarly f2 [1] is a total time at station number 1 of line2, including the entry time e2 and the assemble time a2, 1 at station 1 .

$\left.\begin{array}{l}\mathrm{f} 3[1]=\mathrm{e} 3+\mathrm{a} 3,1 \\ \mathrm{f} 4[1]=\mathrm{e} 4+\mathrm{a} 4,1\end{array}\right\} \quad$ When Type $=2$

If the auto type is 2 then it can be enter either from the line 3 or from the line 4.

f3 [1] is a total time at station number 1 of the line3, including the entry time e3 and the assemble time a3, 1 at station 1. Similarly f4 [1] is a total time at station number 1 of line 4 , including the entry time e 4 and the assemble time a4, 1 at station 1 .

$\left.\begin{array}{l}\mathrm{f} 5[1]=\mathrm{e} 5+\mathrm{a} 5,1 \\ \mathrm{f} 6[1]=\mathrm{e} 6+\mathrm{a} 6,1\end{array}\right\} \quad$ When Type $=3$

If the auto type is 3 then it can be enter either from the line 4 or from the line6.

f5 [1] is a total time at station number 1 of the line5, including the entry time e5 and the assemble time a5, 1 at station 1. Similarly f6 [1] is a total time at station number 1 of line6, including the entry time e6 and the assemble time a6, 1 at station 1 .

All which is discussed in the above is about the base case now the central or core part of this model is given below. First the condition is checked to check that whether the auto part assembling on the station $\mathrm{j}$ is same or not ie. If the type is 1 so the selected lines are either 1 or 2 and the first condition is 
If $(f 1[j] \operatorname{stp}=f 3[j] \operatorname{stp}$ and $f 1[j] \operatorname{stp}=f 5[j] s t p)$

Its mean that the same auto part is assembling on jth station of six lines. So the product can move any of the jth station of six lines. and we compute $\mathrm{f} 1[\mathrm{j}], \mathrm{f} 2[\mathrm{j}], \mathrm{f} 3[\mathrm{j}], \mathrm{f} 4[\mathrm{j}] \mathrm{f} 5[\mathrm{j}]$ and $\mathrm{f} 6[\mathrm{j}]$. And we take minimum of these by using the following formula

$$
\begin{aligned}
& \mathrm{fi}[\mathrm{j}]=\min (\mathrm{f} 1[\mathrm{j}-1]+\mathrm{ai}, \mathrm{j}+\mathrm{t} 1, \mathrm{j}-1,1 \\
& \mathrm{I}==1 \quad \mathrm{f} 2[\mathrm{j}-1]+\mathrm{ai}, \mathrm{j}+\mathrm{t} 2, \mathrm{j}-1,1 \\
& \text { f3 }[\mathrm{j}-1]+\mathrm{ai}, \mathrm{j}+\mathrm{t} 3, \mathrm{j}-1,1 \\
& \mathrm{f} 4[\mathrm{j}-1]+\mathrm{ai}, \mathrm{j}+\mathrm{t} 4, \mathrm{j}-1,1 \\
& \text { f5 [j-1] +ai, }+\mathrm{t} 5, \mathrm{j}-1,1 \\
& \text { f6 [j-1] +ai,j + t6, j-1,1) }
\end{aligned}
$$

$$
\}
$$

When $j>=2$

The second condition for the type 1 is

$$
\text { If }(f 1[j] \operatorname{stp}=f 3[j] s t p \text { and } f 1[j] s t p !=f 5[j] s t p)
$$

Its mean that the same auto part is assembling on jth station of line1, line2, line 3 and line 4 but on the lines $5 \& 6$ the auto part assembling are deferent. So the product can only move of the jth station of line1, line2, line 3 , and line 4 . and we compute $\mathrm{fl}[\mathrm{j}], \mathrm{f} 2[\mathrm{j}], \mathrm{f} 3[\mathrm{j}], \mathrm{f} 4[\mathrm{j}]$ and $\mathrm{f} 5[\mathrm{j}]=\mathrm{f} 6[\mathrm{j}]=\infty$. And we take minimum of these by using the above formula. The third condition for the type 1 is

$$
\text { If (f1[j]stp != f3[j]stp and } f 1[j] s t p=f 5[j] s t p)
$$

Its mean that the same auto part is assembling on jth station of line1, line2, line 5 and line 6 but on the lines $3 \& 4$ the auto part assembling is deferent. So the product can only move of the jth station of line1, line2, line 3 , and line 4 . and we compute $f 1[j], f 2[j], f 5[j], f 6[j]$ and $f 3[j]=f 4[j]=\infty$

The forth condition for the type 1 is

$$
\text { If (f1[j]stp != f3[j]stp and f1[j]stp != f5[j]stp ) }
$$

Its mean that there is no same auto part assemble on jth station of line3, line4, line 5 and line6 so. So the product can only move of the jth station of linel but not others. and we compute $f 1[j], f 2[j]$ and $f 3[j]=f 4[j]=f 3[j]=f 4[j]=\infty$.

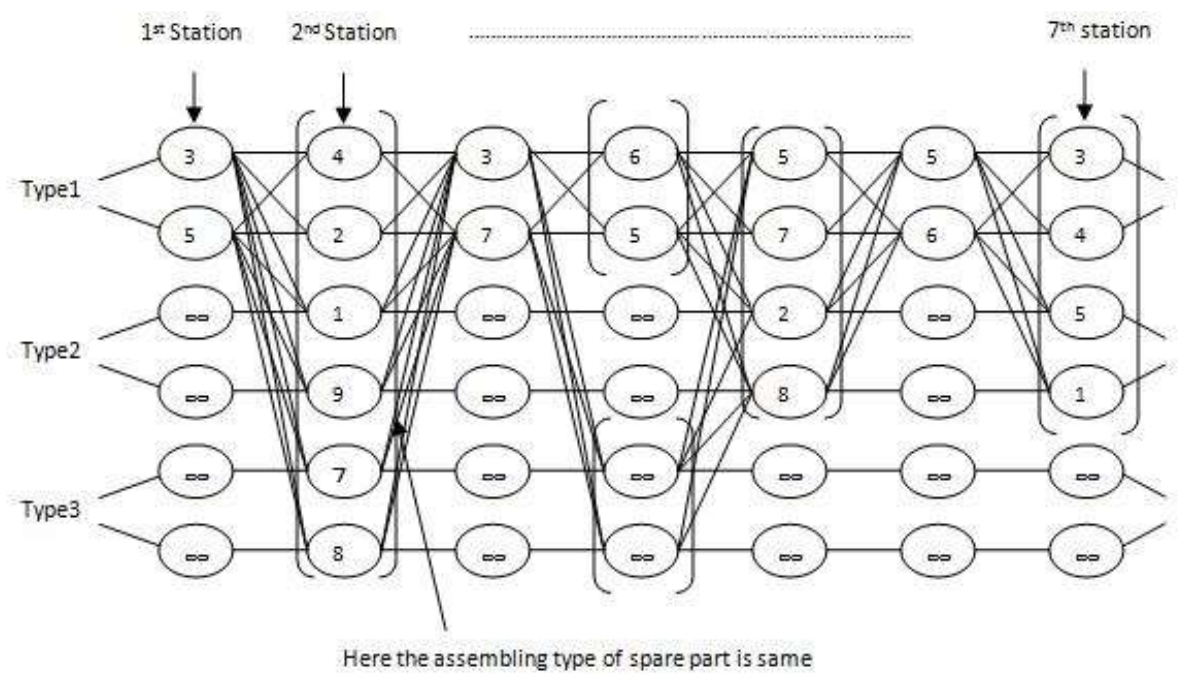

Fig. 3 shows the scenario of the type 1 for all condition. This diagram shows that the same auto parts are assemble at station 2 of the six lines it means that first condition is true and other are false and on the station 3 the forth condition is true and other are false and on the station 4 the third condition is true and other are false and on the station 5 the second condition is true and other are false and then on the $6^{\text {th }}$ station once again the forth condition is true and other are false and on $7^{\text {th }}$ station the second condition is true and other are false.

Similarly when the type is 2 then the auto enter either the line 3 or line 4 the total time at station 1 is calculate by using the base case which is given above. When the stations is or greater than equal to 2 by using the same formula but the conditions are slightly different and first condition is

$$
\text { If }(f 3[j] \operatorname{stp}=f 1[j] s t p \text { and } f 3[j] s t p=f 5[j] s t p)
$$


Its mean that the same auto part is assembling on $\mathrm{j}^{\text {th }}$ station of six lines. So the product can move any of the $\mathrm{j}^{\text {th }}$ station of six lines. and we compute $\mathrm{f} 1[\mathrm{j}], \mathrm{f} 2[\mathrm{j}], \mathrm{f} 3[\mathrm{j}], \mathrm{f} 4[\mathrm{j}] \mathrm{f} 5[\mathrm{j}]$ and $\mathrm{f} 6[\mathrm{j}]$. And we take minimum of these by using the above formula

The second condition for the type 2 is

$$
\text { If }(f 3[j] s t p=f 1[j] s t p \text { and } f 3[j] s t p !=f 5[j] s t p)
$$

Its mean that the same auto part is assembling on $\mathrm{j}^{\text {th }}$ station of line 1 , line 2 , line 3 and line 4 but on the lines $5 \& 6$ the auto part assembling are deferent. So the product can only move of the $\mathrm{j}^{\text {th }}$ station of line 1 , line2, line 3 , and line 4 and we compute $f 1[j], f 2[j], f 3[j], f 4[j]$ and $f 5[j]=f 6[j]=\infty$. And we take minimum of these by using the above formula. The third condition for the type 2 is

$$
\text { If (f3[j]stp != f1[j]stp and f3[j]stp = f5[j]stp ) }
$$

It means that the same auto part is assembling on $\mathrm{j}^{\text {th }}$ station of line 3 , line 4 , line 5 and line 6 but on the lines $1 \& 2$ the auto part assembling is deferent. So the product can only move of the jth station of line3, line4, line5, and line 6 and we compute $f 3[j], f 4[j], f 5[j], f 6[j]$ and $f 1[j]=f 2[j]=\infty$.

The forth condition for the type 2 is

$$
\text { If (f3[j]stp != f1[j]stp and f3[j]stp != f5[j]stp ) }
$$

It means there is no same auto part assembled on $\mathrm{j}^{\text {th }}$ station of line1, line2, line 5 and line 6 so. So the product can only move of the $j^{\text {th }}$ station of line 3 and line 4 but not others. So we compute $f 3[j], f 4[j]$ and $f 1[j]=f 2[j]=f 5[j]=f 6[j]=\infty$.

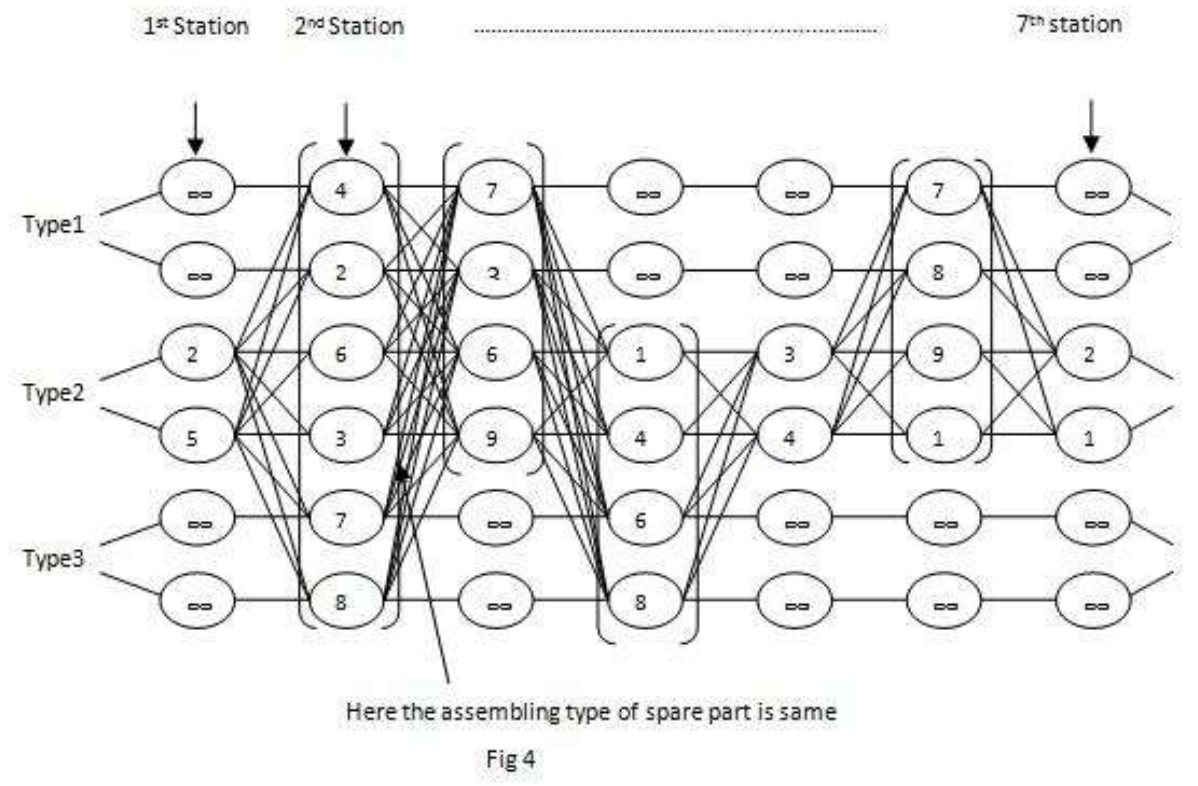

Fig. 4 shows the scenario of the type 2 for all condition. This diagram shows that the same auto parts are assemble at station 2 of the six lines it means that first condition is true and other are false and on the station 3 the second condition is true and other are false and on the station 4 the third condition is true and other are false and on the station 5 the forth condition is true and other are false and then on the $6^{\text {th }}$ station once again the second condition is true and other are false and on $7^{\text {th }}$ station the forth condition is true and other are false.

Similarly when the type is 3 then the auto enter either the lin 5 or line 6 the total time at station 1 is calculate by using the base case which is given above. When the stations is equal to or greater than 2 we use the same formula but the conditions are slightly different and first condition is

$$
\text { If (f5[j]stp }=\mathrm{f} 1[\mathrm{j}] \mathrm{stp} \text { and } \mathrm{f5}[\mathrm{j}] \mathrm{stp}=\mathrm{f3}[\mathrm{j}] \mathrm{stp})
$$

Its mean that the same auto part is assembling on jth station of six lines. So the product can move any of the jth station of six lines. So we compute f1[j], f2[j], f3[j], f4[j] f5[j] and f6[j]. And we take minimum of these by using the above formula. The second condition for the type 3 is

$$
\text { If }(f 5[j] \operatorname{stp}=f 1[j] s t p \text { and } f 5[j] s t p !=f 3[j] s t p)
$$

Its mean that the same auto part is assembling on jth station of line1, line2, line5 and line 6 but on the lines $3 \& 4$ the auto part assembling are deferent. So the product can only move of the jth station of line1, line2, line5, and line6. So we compute $f 1[j], f 2[j], f 5[j], f 6[j]$ and $f 3[j]=f 4[j]=\infty$. And we take minimum of these by using the above formula. The third condition for the type 3 is

$$
\text { If (f5[j]stp != f1[j]stp and f5[j]stp = f3[j]stp ) }
$$


Its mean that the same auto part is assembling on $\mathrm{j}^{\text {th }}$ station of line 3 , line 4 , line 5 and line 6 but on the lines $1 \& 2$ the auto part assembling is deferent. So the product can only move of the jth station of line3, line4, line5, and line 6 and we compute $f 3[j], f 4[j], f 5[j], f 6[j]$ and $f 1[j]=f 2[j]=\infty$.

The forth condition for the type 3 is

$$
\text { If (f5[j]stp != f1[j]stp and f5[j]stp != f3[j]stp ) }
$$

Its mean that there is no same auto part assemble on $\mathrm{j}^{\mathrm{j} \text { th }}$ station of line1, line2, line5 and line6 so. So the product can only move of the $\mathrm{j}^{\text {th }}$ station of line 5 and line 6 but not others.

So we compute f5[j], f6[j] and $f 1[j]=f 2[j]=f 3[j]=f 4[j]=\infty$.

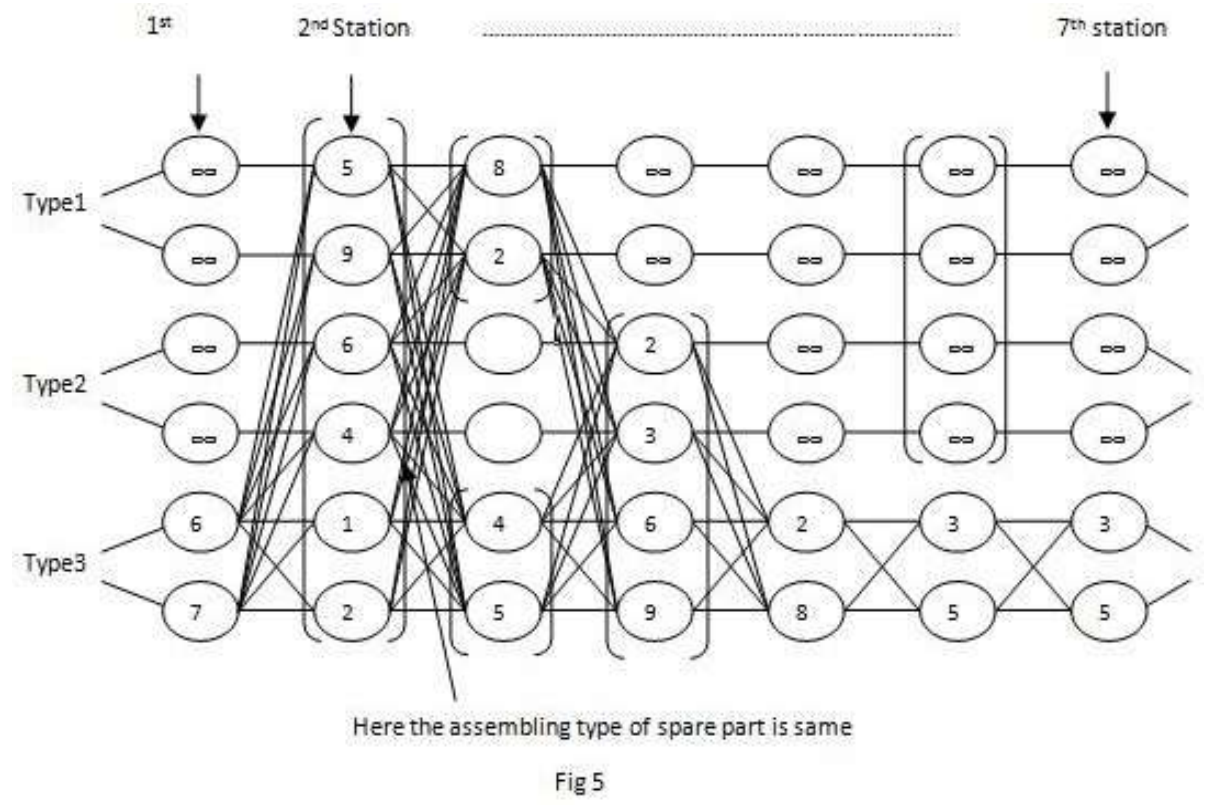

Fig. 5 shows the scenario of the type 3 for all condition. This diagram shows that the same auto parts are assemble at station 2 of the six lines it means that first condition is true and other are false and on the station 3 the forth condition is true and other are false and on the station 4 the third condition is true and other are false and on the station 5 the second condition is true and other are false and then on the $6^{\text {th }}$ station once again the forth condition is true and other are false and on $7^{\text {th }}$ station the second condition is true and other are false.

The fastest time denoted by $\mathrm{f}^{*}$ is.

$$
f^{*}=\min (f 1[n]+x 1, f 2[n]+x 2, f 3[n]+x 3, f 4[n]+x 4, f 5[n]+x 5, f 6[n]+x 6) .
$$

\section{Conclusion and Future Directions}

Assembly lines are widely used in many industries and have a vital role in manufacturing field it is the fastest method of manufacturing products. Before the development of this model when the manufacturers wanted to manufacture more than one type of auto then they couldn't assemble concurrently because they have only two assembly lines which are only for one type of auto, but now after the development of this model it is possible that three type of auto can be assembled concurrently because now we have six assembly lines rather than two lines and may have more than two parallel stations, may be four or six stations are available for assembling. So it is concluded that it is most faster assembling method up to three autos.

There are some restrictions in the proposed model i.e. if the type of auto is 1 then it can only enter either line 1 or line2, if the type is 2 then it can enter either from line 3 or from line 4 and similarly if the type is 3 then it can enter either from line 5 or from line6. This restriction can be taken as future research problem. It is also possible that the model may be developed for more than three type of autos and ' $n$ ' assembly lines. 


\section{REFERENCES}

[1] Bukchin, J., \& Tzur, M. (2000). Design of flexible assembly line to minimize equipment cost. Iie transactions, 32(7), 585-598.

[2] Thomas h. cormen, charles e. leiserson, Ronald 1. rivest, Clifford stein: Introduction to Algorithms, second edition.

[3] Yuanhui Zhang, Peter B. Luh, Kiyoshi Yoneda, Toshiyuki Kano, Yuji Kyoya: Mixed model assembly line scheduling using the lagrangian relaxation technique; Storrs, CT 06269-2157, USA

[4] Levitin, G., Rubinovitz, J., \& Shnits, B. (2006). A genetic algorithm for robotic assembly line balancing. European Journal of Operational Research, 168(3), 811-825.

[5] Müller-Hannemann, M., \& Weihe, K. (2006). Moving policies in cyclic assembly line scheduling. Theoretical computer science, 351(3), 425-436.

[6] Assembly-line scheduling with concurrent operations and parallel machines. INFORMS Journal on Computing,01-JAN-02

[7] Christian Becker, Armin Scholl: A survey on problem and methods in generalized assembly line balancing, 21/2003, ISSN 1611-1311

[8] Van der Zee, D. J., \& Slomp, J. (2005, December). Simulation and gaming as a support tool for lean manufacturing systems: a case example from industry. In Proceedings of the 37th conference on Winter simulation (pp. 2304-2313). Winter Simulation Conference.

[9] Bartholdi, J. J., Eisenstein, D. D., \& Lim, Y. F. (2006). Bucket brigades on in-tree assembly networks. European Journal of Operational Research, 168(3), 870-879.

[10] Hayrinen, T., Johnsson, M., Johtela, T., Smed, J., \& Nevalainen, O. (2000). Scheduling algorithms for computer-aided line balancing in printed circuit board assembly. Production Planning \& Control, 11(5), 497510. 